\title{
Belgeo
}

Revue belge de géographie

1 | 2013

Modelling and benchmarking of borders

\section{Vers un modèle multiscalaire des territoires frontaliers intérieurs à l'Union européenne}

Toward a multiscalar model of border territories in the European Union

\section{Grégory Hamez}

\section{(2) OpenEdition}

\section{Journals}

Édition électronique

URL : http://journals.openedition.org/belgeo/10558

DOI : 10.4000/belgeo.10558

ISSN : 2294-9135

Éditeur :

National Committee of Geography of Belgium, Société Royale Belge de Géographie

Référence électronique

Grégory Hamez, « Vers un modèle multiscalaire des territoires frontaliers intérieurs à l'Union européenne », Belgeo [En ligne], 1 | 2013, mis en ligne le 31 octobre 2013, consulté le 19 avril 2019 URL : http://journals.openedition.org/belgeo/10558; DOI : 10.4000/belgeo.10558

Ce document a été généré automatiquement le 19 avril 2019

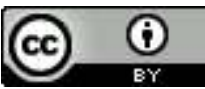

Belgeo est mis à disposition selon les termes de la licence Creative Commons Attribution 4.0 International. 


\title{
Vers un modèle multiscalaire des territoires frontaliers intérieurs à l'Union européenne
}

\author{
Toward a multiscalar model of border territories in the European Union
}

\author{
Grégory Hamez
}

Les conditions d'un accueil en délégation CNRS à l'UMR Géographie-Cités ont permis la réalisation de cet article. Je remercie également les trois relecteurs anonymes pour leurs commentaires sur la première version de l'article.

\section{Introduction}

1 La modélisation des espaces frontaliers se heurte à des difficultés de trois ordres: sémantique, contextuel et conceptuel. Sémantique, car au-delà d'une évidence apparente, la "frontière" et les mots qui en sont dérivés sont porteurs d'un horizon de représentation différent suivant les nations, et font l'objet de stratégies de communication variées concourant à obscurcir leur perception (Foucher, 2007). Contextuel, en raison de l'unicité de chaque frontière dans la singularité des Etats et régions en présence, des vicissitudes historiques qu'ils ont traversées et des processus sociaux singuliers (Bauder, 2011). Conceptuel, en termes d'appareillage théorique permettant de traduire la complexité des processus d'ordre économique, politique, sociétal et culturel se croisant dans ces espaces (Brunet-Jailly, 2005). L'objet de cet article est de contribuer à progresser dans la compréhension des territoires frontaliers, à travers un modèle multiscalaire. En reprenant la définition de Peter Haggett modifiée par François Durand-Dastès, est ici considéré comme modèle "une représentation schématique de la réalité, élaborée en vue de la comprendre et de la faire comprendre" (Durand-Dastès, 2001). La modélisation est d'abord linguistique, dans la mesure où la façon dont nous nous représentons la réalité est étroitement liée au lexique que nous utilisons, qui peut orienter notre perception; elle est ensuite idéelle. Sur la base des écrits théoriques existants, le modèle présenté croise les trois dimensions du territoire que sont 
les pratiques spatiales, les représentations et la gouvernance, avec les trois échelles locale, nationale et européenne. L'article commence par une clarification des mots et des concepts à utiliser pour approcher les espaces en situation frontalière, se poursuit par un exposé du modèle multiscalaire, et se termine en ouvrant quelques pistes de recherche issues $d u$ croisement entre les différentes dimensions et les niveaux d'abstraction du modèle.

\section{Pour une clarification des mots exprimant la frontière et ses processus}

2 Les mots utilisés pour qualifier les phénomènes frontaliers ont évolué au cours des dernières décennies dans l'Union européenne (UE). Premier signe de cette évolution, le mot transfrontalier connaît une inflation d'usage. Il a émergé en 1980 dans la "Convention de Madrid relative à la coopération transfrontalière des collectivités ou autorités territoriales", supplantant les termes alors en cours d'interfrontalier, supra-frontalier ou supra-frontière (Conseil de l'Europe, 1968 ; Wackermann, 1973). Le préfixe trans- ayant les deux sens de traversée d'une part, et de principe supérieur qui s'impose aux parties en présence d'autre part, le terme "transfrontalier" est utilisé à la fois pour qualifier des pratiques et actions nouvelles à travers les frontières (notamment la coopération transfrontalière, les institutions transfrontalières telles que le GECT, ou l'élaboration de stratégies transfrontalières), mais aussi des phénomènes existant de longue date; les travailleurs frontaliers sont ainsi appelés aujourd'hui des travailleurs transfrontaliers, et sont désignés de cette façon dans le Traité CE. Second signe de cette évolution dans l'usage linguistique, les espaces frontaliers intérieurs de l'UE sont souvent associés dans les discours de marketing territorial à une dimension européenne qui leur serait intrinsèque : ce sont des "laboratoires de l'intégration européenne"; les constructions institutionnelles qui tentent de les représenter se dénomment eurorégions; des villes comme Lille utilisent leur situation frontalière pour revendiquer une envergure européenne (Paris \& Mons, 2009).

3 Cette évolution linguistique traduit certes une évolution sémantique, à la mesure des nouvelles réflexions, actions et stratégies qui s'établissent désormais sur une base transfrontalière, comme des politiques européennes attachées aux espaces frontaliers. Elle témoigne aussi d'un esprit du temps particulier, que Michel Foucher dénomme "mode de la rhétorique du sans-frontière" (Foucher, 2007). Elle est questionnable dans la mesure où elle amène à des raccourcis fragiles d'un point de vue scientifique.

4 A titre d'exemple sur les risques que fait courir le flottement terminologique, considérons les deux affirmations suivantes porteuses d'un raisonnement similaire sur le lien entre travail frontalier et intégration européenne, la première émanant d'une étude menée pour le Parlement européen, la seconde d'un article scientifique : "Le travail frontalier (... ) est révélateur des progrès et des limites de la construction européenne dans le cadre de la réalisation de la libre circulation des personnes." (Parlement européen, 1997); "Le robuste processus de décloisonnement découlant de l'intégration européenne pousse à une augmentation du nombre de travailleurs frontaliers" (Sanguin, 2007). Le lien postulé entre l'intégration européenne et le travail frontalier semble a priori logique, en termes de syllogisme et pourrait être reformulé ainsi : l'intégration européenne a instauré une libre-circulation des individus entre les pays membres; or, les travailleurs frontaliers ont 
besoin d'une liberté de circuler à travers les frontières ; donc l'intégration européenne leur permet de circuler et pousse à une augmentation de leur nombre. Cependant, cette assertion ne trouve pas de confirmation dans les faits : l'adhésion d'un pays à l'Union européenne ne provoque pas un afflux de travailleurs frontaliers. C'est même parfois l'inverse comme l'a montré Firmin Lentacker dans le cas de la frontière franco-belge où l'âge d'or du travail frontalier, concomitant des tarifs douaniers, était antérieur à la mise en place de la Communauté économique européenne (Lentacker, 1973). Le travail frontalier répond avant tout à une recherche d'opportunités liée au différentiel binational dans le dynamisme économique, les régimes fiscaux et le coût du travail, ou en termes géographiques dans le jeu entre le type de discontinuité que représente la frontière et l'effet-barrière qu'elle exerce (Grasland, 1997). Dans la mesure où l'intégration européenne favorise à moyen et long terme une convergence économique entre les pays membres, et donc potentiellement une atténuation des discontinuités socio-économiques aux frontières, elle porte même en germe les conditions d'une diminution du travail frontalier, de façon paradoxale au regard du syllogisme rapporté cidessus.

5 Pour éviter que les syllogismes ne débouchent sur des sophismes, il est utile de vérifier si les différents ordres de fait considérés se situent au même niveau d'abstraction, comme le suggère la théorie des types logiques élaborée par Bertrand Russel. En effet, à partir du principe de base qu'une classe ne peut être un membre d'elle-même, ce dernier a montré qu'une hiérarchie existe entre une classe et ses membres - voire entre cette classe et la classe des classes (Russel \& Whitehead, 1910). La confusion survient quand des ordres de faits appartenant à des types logiques différents sont associés. Dans l'exemple précédent, quand le travail frontalier, pratique spatiale dont le ressort tient essentiellement au différentiel salarial et à l'offre de travail, est associé à un principe fondateur de l'Union européenne, la libre circulation, il s'agit bien d'un rapprochement entre des ordres de fait opérant à des niveaux d'abstraction différents. En effet, sauf cas exceptionnel de frontière murée, les populations ont toujours pu franchir les frontières dans leur recherche de travail, et même si le principe de libre-circulation est de nature à faciliter les procédures juridiques liées au travail frontalier il n'agit pas comme élément déclenchant du travail frontalier. Dans une perspective de modélisation des réalités transfrontalières, il s'avère donc essentiel de ne pas confondre les phénomènes se rattachant aux logiques locales, nationales et européennes, dans la mesure où elles se chevauchent à l'endroit des frontières sans nécessairement interférer entre elles.

6 Dans cette recherche des mots justes et de clarification dans le raisonnement, une première étape est de qualifier les espaces situés de part et d'autre de frontières nationales. Les occurrences les plus fréquentes sont espaces transfrontaliers (Moine \& Reitel, 2011) et régions transfrontalières (borderland regions en anglais, Brunet-Jailly, 2005). É voquer un espace transfrontalier permet de rester neutre quant à ses délimitations, ses échelles de pertinence, sa cohérence et sa gouvernance ; la région transfrontalière quant à elle implique une échelle moyenne entre le local et le global, renvoyant aux collectivités territoriales infra-étatiques (Brunet et al., 2005). Cependant, considérer une région transfrontalière comme un agglomérat de différentes régions limitrophes présuppose que cet agglomérat a un sens, ce qui est à démontrer - par exemple, la Grande Région est bien l'association de cinq entités ayant une portée spatiale de taille régionale, mais le pouvoir de décision émanant de cette association demeure faible et les limites de cet ensemble 
sont bien plus vastes que les questions transfrontalières qui s'y posent (Sohn \& Walther, 2009 ; Chilla et al., 2012).

7 Le terme territoire transfrontalier pose également problème, car il tient de l'oxymore: l'idée de territoire demeure liée à celle de l'Etat, tant en langue française qu'en langue anglaise (Brunet, 2005 ; Gottmann, 1973 ; Knippenber \& Mamadouh, 2001), alors que la notion de transfrontalier implique de sortir des limites étatiques. En conséquence, le terme territoire transfrontalier reste rarement utilisé, sauf dans une optique de volontarisme politique en soulignant l'importance d'une gouvernance multi-niveaux pour le rendre opérationnel (Peyrony, 2007). Certes, les dynamiques actuelles remettent en question le lien entre Etat et territoire, et des auteurs plaident pour la complexité croissante de la notion de territorialité sous les effets conjoints de la mondialisation, des transferts de compétence liés à l'intégration européenne et des dynamiques de décentralisation (Jerneck, 2000). Mais si les effets de ces processus globaux ont pu être démontrés pour les grandes métropoles, ils restent à démontrer dans le cas des régions en situation frontalière.

8 La modélisation qui suit ne s'appliquera donc pas à des espaces transfrontaliers pour éviter l'erreur de considérer ces espaces comme des réalités a priori. Elle s'appliquera aux territoires frontaliers, considérés comme parties d'un territoire national contigües de celles d'un autre pays, et pourra éventuellement déboucher sur une analyse en miroir de ces territoires limitrophes. L'objet sera de montrer la dimension multiscalaire de ces territoires, en considérant les échelles spatiales d'analyse comme des types logiques opérant à des niveaux d'abstraction différents.

\section{Une modélisation multiscalaire des territoires frontaliers}

9 Pour définir la nature du territoire frontalier, il faut préalablement définir ce qu'est le territoire. En suivant Maryvone Le Berre et Roger Brunet, le territoire traduit un processus d'appropriation d'une portion de la surface terrestre par un groupe social ; il se lit à travers des pratiques de l'espace qui le façonnent, en mettant en relation les différents lieux; il est l'héritage de structures spatiales passées, mais aussi le fruit des différentes actions de gestion et d'aménagement de cet espace (Le Berre, 1992; Brunet, 1990). Prolongeant cette définition, Alexandre Moine présente le territoire avant tout comme un système, composé de "trois sous-systèmes en interrelation, qui évoluent dans le temps, dans le cadre d'une boucle ininterrompue fondée sur les principes de construction-déconstruction"; ces trois sous-systèmes sont l'espace géographique, approprié, pratiqué et aménagé; les représentations de cet espace; l'ensemble des acteurs agissant sur cet espace. L'intérêt de cette définition est de pouvoir déboucher sur une certaine clarté conceptuelle, puisque les trois composantes du territoire peuvent être regroupées derrière un ensemble de pratiques spatiales, un ensemble de représentations, et enfin une gouvernance. Ces trois composantes constituent la première entrée du modèle.

La seconde entrée du modèle consiste en différentes échelles d'analyse indispensables à la compréhension des territoires frontaliers. La définition de ces territoires ne peut se faire sans référence à plusieurs échelles spatiales, notamment l'échelle nationale de gestion et d'appartenance et l'échelle locale des interactions possibles avec les territoires voisins 
(Guichonnet \& Raffestin, 1974). Le fonctionnement spatial doit se comprendre en termes d'emboîtement d'échelles, par exemple quand une petite ville telle que Comines Belgique est intégrée à l'aire urbaine fonctionnelle frontalière voisine de Lille, mais qu'à plus petite échelle c'est toute une vaste région urbaine qui est engagée dans un processus de rapprochement transfrontalier, de Béthune à Charleroi et de Cambrai à Courtrai (Vandermotten, 2007). Il convient d'y ajouter l'échelle européenne en termes de gouvernance, par l'intermédiaire des financements européens à la coopération ou des instruments communautaires de gestion commune comme les GECT. Dans cette première formulation du modèle, trois échelles seront proposées: l'échelle locale, l'échelle nationale et l'échelle européenne. La détermination de ces échelles s'est faite dans un souci de généralisation possible à tous les territoires frontaliers de l'Union européenne en particulier, il paraît inopportun de considérer une échelle régionale dans la mesure où elle peut se confondre avec l'échelle nationale dans les petits pays ainsi que dans les pays fédéraux de l'UE. Par ailleurs, le choix de considérer une échelle nationale peut sembler surprenant au regard de l'hétérogénéité de taille des pays dans l'UE, mais demeure justifié dans les analyses frontalières puisque l'horizon national demeure structurant tant pour les pratiques spatiales, les représentations et la gouvernance. L'échelle dite locale correspond à des ordres de fait infranationaux.

Chacune des composantes du territoire sera envisagée suivant ces trois échelles. Les pratiques spatiales sont entendues comme les relations de travail, de commerce ou de tourisme/loisir. La question sera de discerner l'ampleur des flux de portée locale, nationale et européenne - et d'apprécier la part des flux transfrontaliers dans cet ensemble. Par exemple, la population de l'aire urbaine lilloise nourrit des relations variées avec les villes belges limitrophes, est également fortement reliée à l'aire parisienne mais beaucoup moins aux pôles européens voisins de Bruxelles et Londres, comme l'indiquent par exemple les chiffres du trafic sur les réseaux ferroviaires à grande vitesse entre ces pôles (Hamez, 2004); les pratiques sont donc à la fois locales transfrontalières et nationales, mais peu européennes. Le cas de Luxembourg-Ville est différent, la présence des institutions européennes rendant l'échelle européenne significative pour certaines pratiques spatiales.

12 En outre, comme les pratiques spatiales transfrontalières sont directement liées aux écarts de développement entre les espaces et à leurs densités de population, leur prise en compte amène d'emblée à distinguer les espaces à fortes relations de l'Europe du nordouest (frontières entre France, Suisse, pays du Benelux, l'Allemagne et ses voisins orientaux) et les espaces où les frontières sont beaucoup moins traversées comme au sud, au nord et à l'est de l'Union européenne.

13 En termes de représentations, les populations des territoires frontaliers seront distinguées suivant qu'elles se sentent appartenir préférentiellement à leur pays, à un espace infranational ou à l'Europe. Les enquêtes de l'Eurobaromètre ont montré que de fortes différences existent entre les pays dans le sentiment d'appartenance à l'Europe ; il serait intéressant de poursuivre l'analyse quant aux perceptions dans les territoires frontaliers.

14 En termes de gouvernance, l'intérêt de distinguer l'horizon européen, national ou infranational est d'apprécier le rôle respectif des trois sphères de décision, et d'identifier par exemple les territoires où la décision se prend principalement à l'interface entre acteurs locaux et instruments européens, des territoires où l'Etat reste prépondérant 
dans les instances de décision. L'horizon européen correspond à l'importance du recours aux instruments européens de coopération tels qu'Interreg.

Ce modèle permet de croiser des ordres de fait opérant à des échelles différentes, n'ayant pas nécessairement de lien entre eux mais concourant à caractériser le territoire frontalier. Il doit être spatialisé pour que ce croisement prenne tout son sens. En effet, il serait réducteur de chercher une valeur moyenne pour les différentes composantes du territoire et leurs échelles, sans tenir compte de la distance à la frontière. Il a été montré que la portion d'espace directement concernée par les échanges frontaliers dans leur quotidienneté était réduite à un ourlet frontalier dont la largeur n'excède pas 5 à 10 kilomètres, l'ampleur des échanges se réduisant nettement au-delà de cette limite (Buxeda, 2006, pour la frontière franco-allemande). Le tableau 1 et la figure 1 donnent un exemple théorique de cette spatialisation.

Tableau 1. Les composantes du territoire et leurs échelles.

\begin{tabular}{|l|c|c|c|}
\hline Échelles & locale & nationale & européenne \\
\hline Pratiques spatiales & $\Delta$ & & \\
\hline Représentations & 0 & & \\
\hline Gouvernance & $\square$ & \\
\hline
\end{tabular}

16 Le modèle entend montrer la grande variété possible des cas de figure, dans la mesure où les différentes catégories et échelles ne s'excluent pas mais peuvent se superposer. Le sentiment d'appartenance d'une population frontalière peut très bien être principalement national, mais aussi local et européen. Des pratiques spatiales intenses sur une base locale transfrontalière n'excluent pas d'autres pratiques tout aussi fortes sur une base nationale, et un sentiment d'appartenance national. 
Figure 1. Un modèle multiscalaire appliqué à un territoire frontalier fictif.

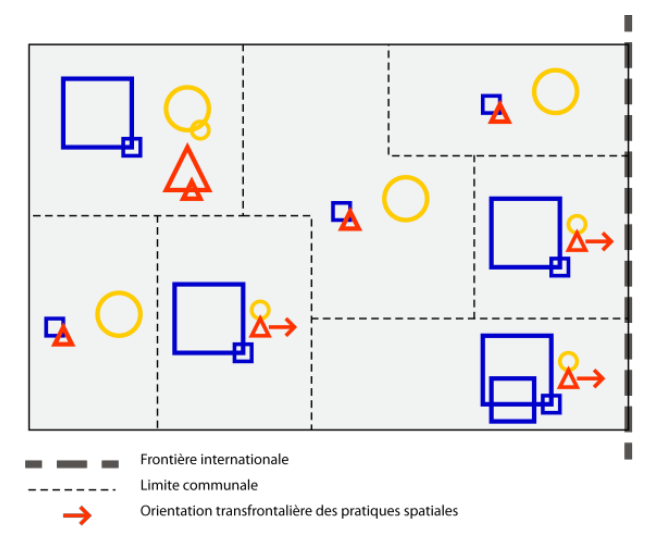

A titre d'exemple, voici quelques caractéristiques possibles de ces communes:

Commune frontalière à l'́cart des relations transfrontalières, pour laquelle le

प.

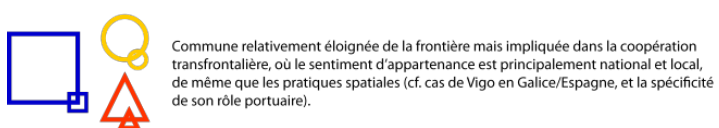
de son rôle portuaire.
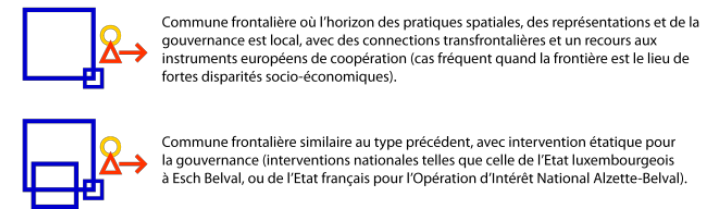

Le modèle permet une première étape conceptuelle, qui ouvre sur une seconde étape d'opérationnalisation. L'opérationnalisation suppose de répondre à un ensemble de questions méthodologiques sur la mesure des représentations, des échelles de la gouvernance et des pratiques spatiales, ce qui soulève les enjeux techniques de la définition des données et de l'unité spatiale modifiable (de Ruffray \& Hamez, 2011). En effet, la production statistique demeurant une prérogative étatique et non communautaire, les modalités de recensement diffèrent suivant les pays de même que le maillage spatial dans lequel sont proposées les données. L'existence d'Eurostat ne doit pas faire illusion en la matière dans la mesure où l'agence européenne travaille sur des données de seconde main, collectant et s'efforçant d'harmoniser des données produites nationalement. La constitution de bases de données transfrontalières est donc un premier défi.

En parallèle à la constitution de cette base de données, l'opérationnalisation doit passer par des enquêtes auprès de publics différents permettant d'apprécier les représentations et la gouvernance. L'objet de cet article n'est pas de définir un protocole méthodologique figé, ce qui serait un exercice peu fécond au regard de la variabilité des contextes frontaliers. Il est bien davantage de montrer qu'au-delà de cette variabilité, des progrès substantiels peuvent être faits dans la compréhension de la complexité frontalière à travers le double niveau de lecture présenté dans la figure 1. Son intérêt est également de servir de guide pour ouvrir de nouvelles perspectives de recherche. 


\section{La vocation heuristique du modèle}

Des problématiques et orientations de recherche originales peuvent découler du modèle en articulant différentes composantes du territoire et différentes échelles. Les trois questions qui suivent sont données à titre d'exemple ; elles ont été choisies en ce qu'elles articulent thématiques et échelles spatiales distinctes, et qu'elles ouvrent des champs de recherche encore peu exploré. La première rapproche le sentiment d'appartenance européen avec les pratiques locales transfrontalières; la deuxième la gouvernance européenne avec les pratiques locales; la troisième les différentes composantes et échelles du territoire à travers le concept global de cohésion territoriale.

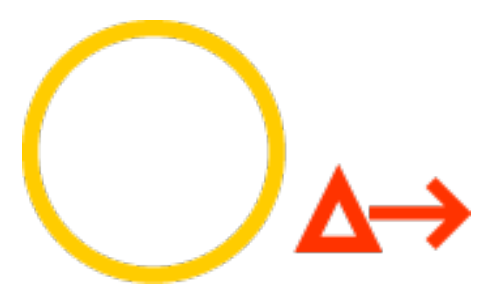

Première question, y a-t-il une relation entre des pratiques spatiales locales transfrontalières et un sentiment d'appartenance européen? Cette question permet d'élargir l'acception du syllogisme rapporté au début de cet article. En effet, si le travail frontalier et la perception de l'Europe appartiennent à des niveaux d'abstraction différents, d'autres facteurs interfèrent comme les discours et pratiques d'une gouvernance transfrontalière et peuvent concourir à l'émergence de représentations particulières. De nombreux travaux ont déjà abordé la perception que les populations frontalières ont de leur espace de vie (par exemple Strüver, 2005), beaucoup moins la perception qu'elles ont de l'Europe, en dehors d'une recherche à caractère anthropologique menée de part et d'autre de l'ancien Rideau de fer (Armbruster et al., 2003). Dans ce dernier cas, par l'intermédiaire d'une analyse des discours frontaliers, il est ressorti que ni "l'Europe" ni "l'européanité" n'étaient choisies comme catégories d'identification, comme si le contexte local et le poids de l'histoire importaient davantage que le processus de construction de l'identité européenne. La question demeure entière dans le cas des frontières entre pays de l'Europe de l'ouest où le processus de construction européenne est plus ancien, et où les frontières n'ont jamais été complètement imperméables.
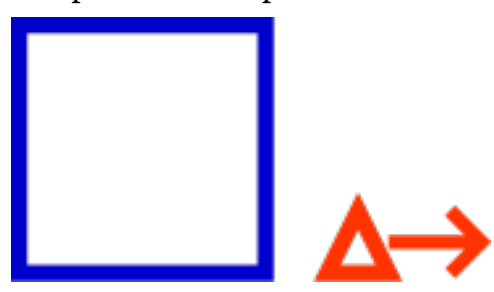

21 Deuxième question, y a-t-il une adéquation entre les relations fonctionnelles locales transfrontalières et les actions mises en place par les outils communautaires de coopération transfrontalière? Suivant cette approche fonctionnaliste, et sans attendre une adéquation rigoureuse entre le fonctionnel et la gouvernance, il s'agit de signaler les déconnections fortes entre pratiques transfrontalières et grandes actions de la gouvernance car de telles situations peuvent déboucher sur des représentations particulières. A titre d'exemple, et dans la mesure où un des objectifs d'Interreg est de 
créer un marché du travail intégré entre les deux côtés des frontières, une corrélation peut être recherchée entre l'intensité du travail frontalier dans les territoires, et sa prise en compte dans les projets Interreg ${ }^{1}$. En effet, comme le travail est une dimension importante de la vie des populations, si ces dernières traversent massivement la frontière pour le travail mais que les actions de coopération transfrontalière concernent peu le travail, il s'ensuit une déconnection qui peut nourrir une représentation de l'Europe éloignée des besoins des citoyens. Ainsi, le rapprochement des flux de travail frontalier à la frontière nord-est de la France avec les projets de coopération relatifs au travail dans les quatre zones de coopération concernées, permet de mettre en évidence un dysfonctionnement dans le cas de la Grande Région, emblématique de la force du travail frontalier, mais où il n'y a que $11 \%$ des projets Interreg qui traitent du phénomène - trois fois moins que dans le programme Interreg franco-suisse alors que le travail frontalier y revêt une importance similaire (figure 2$)^{2}$. La perception de l'Union européenne en seraitelle affectée?

Figure 2. Le travail frontalier à la frontière nord-est de la France, et sa prise en compte dans les projets Interreg.

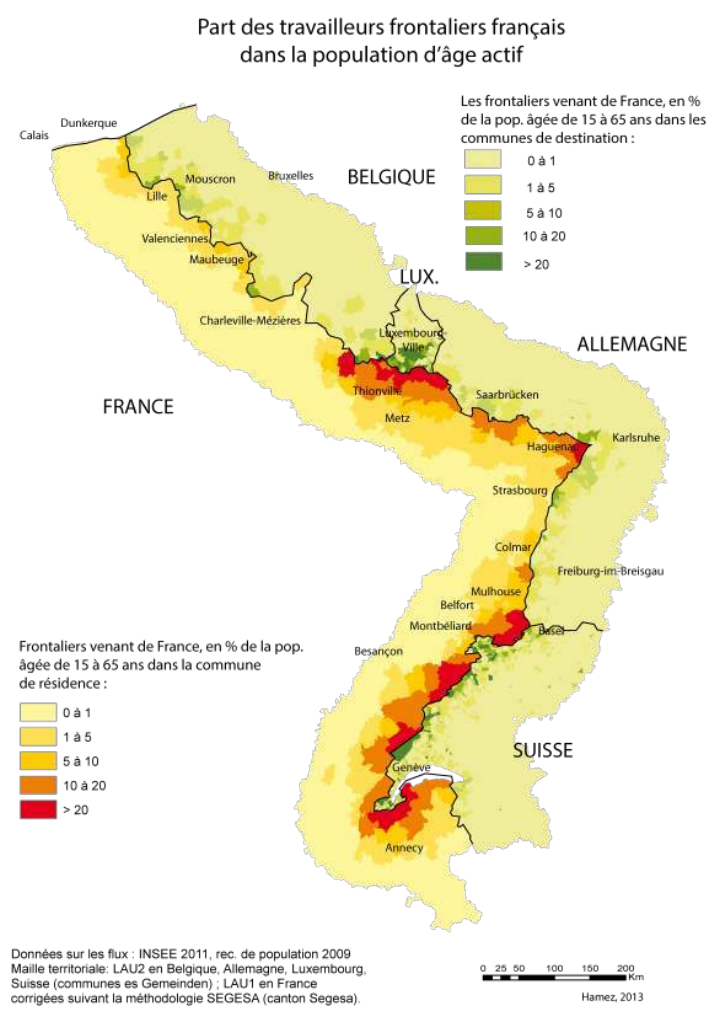


La coopération transfrontalière portant sur le travail frontalier

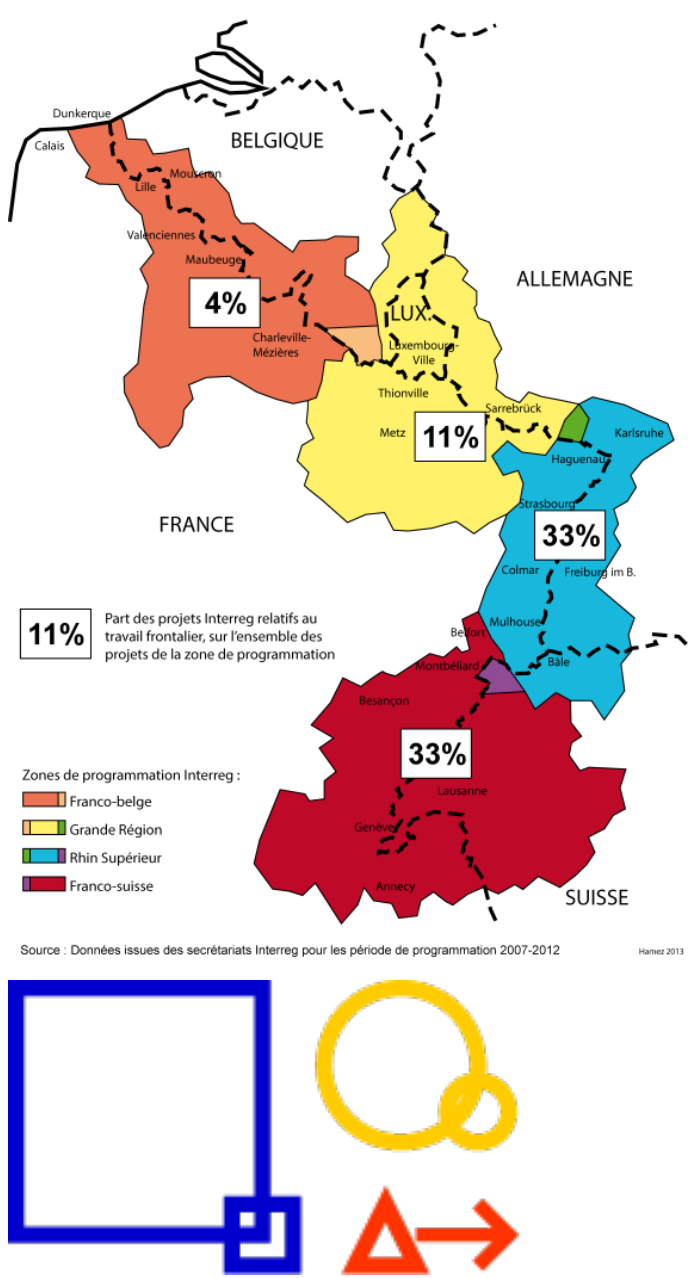

Troisième question, comment appliquer dans le cadre transfrontalier les grands concepts globaux d'aménagement comme la cohésion territoriale, qui transcendent les composantes du territoire et les échelles ? La cohésion territoriale est un des objectifs politiques de l'UE, récemment inscrit dans le traité, et relayé dans les documents d'aménagement de la plupart des pays membres y compris dans les documents régionaux (ESPON CaDEC 2013). Si sa définition est encore en devenir, il est reconnu qu'au niveau des régions de l'UE elle porte l'idée d'une lutte contre les disparités tout en respectant la diversité des territoires, et qu'au niveau local elle a trait à l'accessibilité des habitants aux services. La déclinaison de ce concept dans les territoires frontaliers amène alors à s'interroger sur les écarts régionaux de développement et leur mesure (Grasland \& Hamez, 2004) ainsi qu'à l'échelle locale aux bénéfices que les habitants pourraient retirer d'une meilleure coordination des services publics (de Ruffray \& Hamez, 2009). Regarder ce concept par le prisme du modèle permet de sonder la faisabilité d'une telle coordination : en termes de représentation, les acteurs concernés sont-ils prêts à bâtir un espace transfrontalier des services, qu'il s'agisse des acteurs institutionnels ou des citoyens appelés à utiliser ces services ? En termes de gouvernance, jusqu'où peut-on aller avec les outils existants de la coopération? 


\section{Conclusion} compréhension des processus qui s'y jouent. Par une attention portée aux dimensions du
territoire et aux échelles constitutives du transfrontalier, il permet un regard critique sur ces processus en évitant de prendre pour acquise une réalité transfrontalière postulée dans les discours. Un second intérêt du modèle serait d'être appliqué, et de déboucher sur une typologie des territoires frontaliers. L'enjeu d'une telle entreprise est d'une part scientifique pour faire progresser la connaissance sur ces territoires complexes, d'autre part opérationnel pour adapter les outils de la coopération transfrontalière en fonction du profil des territoires, au lieu de promouvoir la coopération dans tous les contextes transfrontaliers comme c'est le cas actuellement - la coopération peut très bien être de peu d'intérêt dans certains contextes. En effet, les études sur les frontières sont la plupart du temps cloisonnées en approches distinctes; il est rare de considérer à la fois les questions de gouvernance, de représentation et de pratiques spatiales, qui plus est à différentes échelles d'analyse. Il en ressort une connaissance parcellaire des dynamiques frontalières. Pour dépasser cette limite scientifique, il faudrait d'une part inciter les recherches pluridisciplinaires sur les frontières, d'autre part favoriser la constitution de données transfrontalières permettant d'établir un diagnostic. Or, la production de statistiques demeure à l'heure actuelle une prérogative nationale, et les données ne sont pas construites de façon harmonisée ce qui rend difficile toute analyse à grande échelle, requise pour les diagnostics transfrontaliers. Combien de temps encore l'Union européenne pourra-t-elle continuer à mettre en place des politiques sans se donner les moyens d'une véritable analyse territoriale comparative? Par son étymologie, le terme statistique renvoie à l'Etat, par son histoire il renvoie aux grands moments de constitution étatique avec la mise en place de nouvelles nomenclatures, d'un système de poids et mesures comme la Révolution française de 1789 s'y est employée (Desrosières, 2010). Le remplacement des instituts nationaux de statistique par une agence européenne serait à cet égard révolutionnaire en Europe et permettrait d'envisager d'une façon beaucoup plus concrète les processus transfrontaliers ; mais cela supposerait que l'UE soit animée par un grand dessein politique, qui lui fait défaut à ce jour.

\section{BIBLIOGRAPHIE}

ARMBRUSTER H., ROLL C., MEINHOF U.H. (2003), “Imagining Europe: everyday narratives in European border communities", Journal of Ethnic and Migration Studies, 29, 5, pp. 885-899.

BAUDER H. (2011), "Toward a Critical Geography of the Border: Engaging the Dialectic of Practice and Meaning", Annals of the Association of the American Geographers, 101, 5, pp. 1126-1139.

BRUNET R. (1990), Le territoire dans les turbulences, Montpellier, Fayard/Reclus. 
BRUNET R. et al. (2005), Les mots de la géographie : Dictionnaire critique, Paris, Reclus/ La documentation française.

BRUNET-JAILLY E. (2005), “Theorizing borders: An interdisciplinary perspective”, Geopolitics, 10, pp. 643-659.

BUXEDA C. (2006), Intégration territoriale transfrontalière et apprentissage de l'allemand dans le grand Est français, Thèse de géographie, Université Paris 1.

CHILLA T., EVRARD E., SCHULZ C. (2012), “On the territoriality of cross-border cooperation: 'Institutional mapping' in a multi-level context”, European Planning Studies, 20, 6, pp. 961-980

COMMISSION EUROPEENNE (2004), “Communication de la commission aux Etats-Membres, fixant des orientations pour une initiative communautaire concernant la coopération transeuropéenne et destinée à favoriser un développement harmonieux et équilibré du territoire européen INTERREG III", JOEU du 10/09/2004, C226, pp. 2-27.

CONSEIL DE L'EUROPE (1968), Aménagement du territoire, problème européen : rapport de l'assemblée consultative, Bruxelles, Conseil de l'Europe, $145 \mathrm{p}$.

DE RUFFRAY S., HAMEZ G. (2009), “La dimension sociale de la cohésion territoriale : L'exemple de l'accessibilité aux maternités dans la Grande Région”, L'Espace Géographique, 4, pp. 328-344

DE RUFFRAY S., HAMEZ G. (2011), Enjeux des territoires frontaliers à l'échelle nationale: Vers la détermination et la délimitation de pôles transfrontaliers, étude pour l'UMS RIATE et la DATAR, http:// www.ums-riate.fr/documents/rapportDATAR_mai2011.pdf (consulté le 31 mars 2013).

DESROSIERES A. (2010), La politique des grands nombres, Paris, La découverte.

DIDELON C. et al. (2011), "Variations in the scale of feeling and belonging”, Eurobroadmap working paper, 3, 30 p., http://halshs.archives-ouvertes.fr/halshs-00654524/fr/ (consulté le 10/04/2013).

DURAND-DASTES F. (2001), "Les concepts de la modélisation en analyse spatiale", in SANDERS L., Modèles en analyse spatiale, Paris, Hermès Science Publications, pp. 31-60.

FOUCHER M. (2007), L'obsession des frontières, Paris, Perrin.

ESPON projet CaDEC (2013), Capitalisation and Dissemination of ESPON Concepts, rapport final.

ESPON projet TERCO (2012), European Territorial Cooperation as a Factor of Growth, Jobs and Quality of Life, rapport final, $81 \mathrm{p}$.

GOTTMANN J. (1973), The significance of territory, Charlottesville, University Press of Virginia.

GRASLAND C. (1997), À la recherche d'un cadre théorique et méthodologique pour l'étude des maillages territoriaux, Communication aux entretiens Jacques Cartier, "Les découpages du territoire", Lyon, 12/1997, http://www-census.ined.fr/debat/Contributions/Avant-Fevrier-1999/Grasland-2.html, (consulté le 29 mars 2013).

GRASLAND C., HAMEZ G., 2005, "Vers la construction d'un indicateur de cohésion territoriale européen ?” L’Espace Géographique, 2, pp. 97-116.

GUICHONNET P., RAFFESTIN C. (1974), Géographie des frontières, Paris, PUF.

HAMEZ G. (2004), Du transfrontalier au transnational : Approche géographique. L'exemple de la frontière franco-belge, thèse de géographie, Université Paris 1, http://tel.ccsd.cnrs.fr/documents/ archives0/00/00/71/91/index_fr.html

JERNECK M. (2000), “Europeanization, territoriality and political time”, in HARMSON R., THOMAS W. (eds.), Europeanization: Institution, identities and citizenship, 14, Amsterdam, Yearbook of European Studies, pp. 27-49. 
KNIPPENBERG H., MAMADOUH V. (2001), "State territoriality and beyond: An introduction", Tijdschrift voor economische en sociale geografie, 92, 4, pp. 391-393.

LE BERRE M. (1992), “Territoires”, in BAILLY A. et al., Encyclopédie de la géographie, Paris, Economica.

LENTACKER F. (1973), La frontière franco-belge. Etude géographique des effets d'une frontière nationale sur la vie des relations, thèse de géographie, Université Paris 4.

PARIS D., MONS D. (2009), Lille Métropole, laboratoire du renouveau urbain, Marseille, Parenthèses.

PARLEMENT EUROPEEN, Direction générale des études, (1997), "Les travailleurs frontaliers dans l'Union européenne”, Série Affaires sociales W-16, PE166-692, http://www.europarl.europa.eu/ workingpapers/soci/pdf/w16_fr.pdf (consulté le 16 mars 2013).

PEYRONY J. (2007), “L'aménagement du territoire transfrontalier : retour d'expérience”, Mosella, 32, 1-4, pp. 169-180.

SANGUIN A.-L. (2007), "Les nouvelles perspectives frontalières de l'union européenne après l'élargissement de 2004”, L'Espace Politique, 1, http://espacepolitique.revues.org/index437.html (mis en ligne le 16 janvier 2007 ; consulté le 29 mars 2013).

SOHN C., WALTHER O. (2009), "Métropolisation et intégration transfrontalière : le paradoxe luxembourgeois", Espaces et Sociétés, 3, 138, pp. 51-67.

STRÜVER A. (2005), Stories of the "Boring Border". The Dutch-German Borderscape in People's Minds, Forum Politische Geographie, 2.

VANDERMOTTEN C. (2007), "Les aires urbaines transfrontalières en Europe”, Mosella 32, 1-4, pp. 37-47.

WACKERMANN G. (1973), Les loisirs de l'espace rhénan, de la région zurichoise à la frontière germanonéerlandaise, thèse de géographie, Université Louis Pasteur de Strasbourg.

WHITEHEAD A.N, RUSSEL B. (1910), Principia Mathematica, 3 vol. , $2^{\mathrm{e}}$ éd., Cambridge, Cambridge University Press.

\section{NOTES}

1. Parmi les neuf priorités du programme Interreg, la troisième est formulée ainsi : "Favoriser l'intégration du marché du travail et l'inclusion sociale", et décrite comme: "Création et/ou développement d'un marché du travail intégré de part et d'autre de la frontière; (...) amélioration du fonctionnement du marché du travail transfrontalier" (Commission européenne, 2004).

2. La "Grande Région" désigne le regroupement institutionnel des entités suivantes : le Grand Duché du Luxembourg, la Wallonie (Belgique), la région Lorraine (France), les Länder de Rhénanie-Palatinat et de Sarre (Allemagne). Les données sur le travail frontalier proviennent du recensement français de la population (2009) ; les projets Interreg sont ceux de la période de programmation 2007-2012, et les informations proviennent des secrétariats Interreg. Les projets ont été dépouillés en suivant les thèmes proposés dans le projet ESPON TERCO (2012). Les résultats de cette analyse ont été présentés au colloque "Unpacking Cross-Border Governance", Luxembourg, septembre 2012. 


\section{RÉSUMÉS}

L'article propose un modèle théorique multiscalaire des territoires frontaliers dans l'Union européenne, en s'appuyant sur une définition du territoire englobant les trois dimensions des pratiques spatiales, des représentations et de la gouvernance, et en considérant trois échelles spatiales d'analyse pour interpréter les logiques transfrontalières (locale, nationale, européenne). Ce modèle ouvre des perspectives de recherche pour appréhender la complexité des dynamiques frontalières en termes de déconnection entre les échelles spatiales de manifestation des phénomènes, ainsi qu'entre les niveaux territoriaux d'action.

The paper proposes a theoretical multiscalar model of border territories in the European Union, based on a definition of territory encompassing the three dimensions of the spatial practices, representations and governance, through the lens of three spatial scales to interpret the cross border functioning (local, national, European). The model opens up research perspectives to grasp the complexity of border processes in terms of disjunction between the spatial scales of phenomena occurrences, as well as between the territorial action levels.

INDEX

Mots-clés : frontière, territoire, transfrontalier, échelle, modèle, Union européenne Keywords : border, territory, cross-border, scale, model, European Union

\section{AUTEUR}

\section{GRÉGORY HAMEZ}

LOTERR, Université de Lorraine, UFR SHA, île du Saulcy, BP30309, 57006 METZ, gregory.hamez@univ-lorraine.fr 